\title{
Early brain-body impact of emotional arousal
}

\author{
Fabien D'Hondt ${ }^{1,2}$, Maryse Lassonde ${ }^{3,4}$ *, Olivier Collignon ${ }^{3,5}$, Anne-Sophie Dubarry $^{3}$, Manon Robert ${ }^{3}$, \\ Simon Rigoulot ${ }^{1,2}$, Jacques Honoré ${ }^{1,2}$, Franco Lepore ${ }^{3,4}$ and Henrique Sequeira ${ }^{1,2,6}$ \\ 1 Université de Lille Nord de France, Lille, France \\ 2 Neurosciences Fonctionnelles et Pathologies, Centre National de la Recherche Scientifique, Université de Lille 2, Lille, France \\ 3 Centre de Recherche en Neuropsychologie et Cognition, Université de Montréal, Montreal, QC, Canada \\ ${ }^{4}$ Centre de Recherche, Centre Hospitalier Universitaire de Sainte-Justine, Montreal, QC, Canada \\ ${ }_{5}^{5}$ Laboratoire de Génie de la Réhabilitation Neurale, Institut des Neurosciences, Université Catholique de Louvain, Brussels, Belgium \\ 6 Neurosciences, Université de Lille 1, Lille, France
}

\section{Edited by:}

Olaf Blanke,

Ecole Polytechnique Fédérale de

Lausanne, Switzerland;

University of Geneva, Switzerland

Reviewed by:

Jane Aspell,

Ecole Polytechnique Fédérale de

Lausanne, Switzerland

Thomas Koenig,

University Hospital of Psychiatry,

Switzerland

${ }^{*}$ Correspondence:

Maryse Lassonde,

Département de Psychologie,

Université de Montréal, Centre de

Recherche en Neuropsychologie et

Cognition, 90 Vincent d'Indy, CP 6128,

succ. Centre-Ville, Montréal, Québec

H3C 3J7 Canada.

e-mail:maryse.lassonde@umontreal.ca
Current research in affective neuroscience suggests that the emotional content of visual stimuli activates brain-body responses that could be critical to general health and physical disease. The aim of this study was to develop an integrated neurophysiological approach linking central and peripheral markers of nervous activity during the presentation of natural scenes in order to determine the temporal stages of brain processing related to the bodily impact of emotions. More specifically, whole head magnetoencephalogram (MEG) data and skin conductance response (SCR), a reliable autonomic marker of central activation, were recorded in healthy volunteers during the presentation of emotional (unpleasant and pleasant) and neutral pictures selected from the International Affective Picture System (IAPS). Analyses of event-related magnetic fields (ERFs) revealed greater activity at $180 \mathrm{~ms}$ in an occipitotemporal component for emotional pictures than for neutral counterparts. More importantly, these early effects of emotional arousal on cerebral activity were significantly correlated with later increases in SCR magnitude. For the first time, a neuromagnetic cortical component linked to a well-documented marker of bodily arousal expression of emotion, namely, the SCR, was identified and located. This finding sheds light on the time course of the brain-body interaction with emotional arousal and provides new insights into the neural bases of complex and reciprocal mind-body links.

Keywords: emotion, brain-body, arousal, magneto-encephalography, skin conductance response, principal component analysis

\section{INTRODUCTION}

The classical approach to mind-body interactions considers that psychological processes modulate general health and physical disease. An integrative neurophysiological approach whereby central and peripheral markers of nervous activity are recorded could therefore help unveil the mechanisms linking brain and body in connection with a specific mental state, and this information could be used to improve health outcomes (Critchley, 2009; Lane et al., 2009). Thus, emotion appears to be a key link between mental states and physical disease (Lane et al., 2009).

This assumption first appeared in the late nineteenth century following the well-known debate between the James-Lange and Cannon-Bard theories of emotion. The James-Lange theory proposes that emotional stimuli first induce peripheral physiological variations, which occur without consciousness of affect. These bodily responses are further interpreted by the brain to produce the feeling state of an emotion (Critchley, 2009). In contrast, the Cannon-Bard theory states that the perception of emotional stimuli evokes brain responses that simultaneously but separately induce bodily responses on the one hand and subjective feeling on the other (Friedman, 2009). From this debate have emerged fundamental questions about the time course of brain and body responses to emotion as well as their role in generating feelings.
Although the James-Lange theory and its temporal aspects are counterintuitive and hardly testable, the debate it has generated versus the Cannon-Bard theory has influenced research on emotion (Lang, 1994). Importantly, a recent study suggested that peripheral physiological responses can occur before the feeling of self-generated emotions, providing support to reconsider the James-Lange theory (Damasio et al., 2000). Damasio proposed a distinction between emotions and feelings whereby emotions are "collections of responses" corresponding to external and measurable reactions expressed via the musculoskeletal system as well as internal and measurable reactions of neurovegetative, neurohormonal, and neuroimmune systems controlled by the central nervous system (Damasio, 2000). Feelings, however, correspond to the subjective experience of these emotional responses (Damasio, 1999). Damasio's theory states that a subjective feeling emerges through the integration of these peripheral and central components of nervous activity. Emotional stimuli are processed by the anterior affective structures, including the amygdala, the temporal pole, the orbitofrontal cortex, and the ventromedial prefrontal cortex (Rudrauf et al., 2009). In these cerebral areas, amygdala activation causes bodily reactions that are mediated by changes in autonomic nervous activity (see Davis and Whalen, 2001 for a review). These body signals (or somatic markers) in turn inform the brain about changes in the internal environment, by means 
of a "body-loop" involving the medial prefrontal cortices, which consider these somatic markers and select the appropriate behavior in response to the environmental stimulation. Nevertheless, somatic markers can also represent the expected body reaction, involving cerebral somatosensory maps, and can more rapidly and efficiently inform the brain for decision-making purposes than the cognitive processes can (Damasio et al., 1991; Damasio, 1994, 1996, 1999).

In recent decades, the central and peripheral components of emotional processing have been extensively examined, in particular using emotional scenes taken from the International Affective Picture System (IAPS, Lang et al., 2005). This system is based on a model of emotions proposed by Lang in which emotions are defined as a function of two main dimensions: (1) arousal, which indexes the level of intensity of a given emotion, whether pleasant or unpleasant, and (2) valence, or the level of pleasantness/unpleasantness experienced (Lang et al., 1993). In response to IAPS pictures, neuroimaging studies have typically found activation in the amygdala (Lane et al., 1999; Paradiso et al., 1999; Liberzon et al., 2000, 2003; Taylor et al., 2003; Phan et al., 2004; Sabatinelli et al., 2005; Britton et al., 2006; Kensinger and Schacter, 2006) and in the medial prefrontal cortices (Lane et al., 1997, 1999; Taylor et al., 2003; Anders et al., 2004; Phan et al., 2004; Britton et al., 2006; Grimm et al., 2006; Kensinger and Schacter, 2006). At the body level, studies on the peripheral impact of emotions have shown that these emotional pictures provoke changes in autonomic activity, reflected in different physiological indices (Lang et al., 1993; Bradley et al., 2001a). In particular, skin conductance response (SCR) is central to Damasio's somatic marker hypothesis (Critchley, 2009). During emotional stimulation, SCR amplitude increases with the subjective assessment of the emotional arousal of the stimulus, regardless of valence (Lang et al., 1993; Bradley et al., 2001a).SCRs therefore constitute a reliable autonomic marker of central activation, indexing emotional arousal and its somatovisceral impact (Sequeira et al., 2009).

We hypothesized that if body responses are involved in generating emotional feelings, this process would require detecting the emotion regardless of the valence of the visual stimulus. This emotional detection would occur at the early stages of affective visual processing, before consciousness of affect. This raises the question of the activity time course in brain areas involved in affective visual processing and the associated bodily responses. Although this information could be very useful in determining brain-body interactions during emotional processing, only rarely (e.g., neuroimagery: Fredrikson et al., 1998; Critchley et al., 2000; Liberzon et al., 2000; Williams et al., 2001; Anders et al., 2004; electrophysiology: Amrhein et al., 2004; Keil et al., 2008) have researchers concomitantly recorded central and peripheral measures of nervous activity. Importantly, only a few studies have used a high temporal resolution functional method to explore cerebral activity. For instance, Rudrauf et al. (2009) observed that early cortical responses were stronger for unpleasant stimuli, while heart beats in the first $500 \mathrm{~ms}$ post stimulus showed longer intervals for unpleasant than for neutral stimuli relative to the preceding beat. However, although their study explored the temporal order of brain and body responses to emotional stimulation, it did not directly correlate these responses. Moreover, the pattern of heart rate fluctuations in response to emotional stimulation presents a more complex association with affective reports than with SCRs, and appears to be affected by both emotional dimensions, namely arousal and valence (Lang et al., 1993; Bradley et al., 2001a; Critchley, 2009). Finally, the relative contributions of the sympathetic and parasympathetic nervous systems to the heart rate fluctuations observed during emotional processing are unclear (Lang et al., 1993; Bradley et al., 2001a; Ribeiro et al., 2007; Sequeira et al., 2009). Consequently, the aim of the current study was to use a high temporal resolution functional method with fairly good spatial resolution to determine the temporal stages of brain processing that are specifically related to the bodily impact of emotions, indexed by a robust peripheral marker of emotional activation. Hence, we used SCRs as they reflect the specific responses of the sympathetic nervous system to the arousal dimension of emotional processing, without direct influence of the parasympathetic system (Critchley, 2009; Sequeira et al., 2009). Thus, we combined for the first time recordings of whole head magneto-encephalogram (MEG) data with SCRs during the presentation of emotional and neutral IAPS pictures in healthy human adults.

\section{MATERIALS AND METHODS SUBJECTS}

Eighteen healthy right-handed (Oldfield, 1971) students (11 females; mean $25.9 \pm 8.5$ years) were included in the study. All participants had normal or corrected to normal vision and had never presented neurological or psychiatric disorders. Participants gave their written informed consent for the protocol, which was approved by the ethics committee of the Universite de Montréal. The study was conducted at the MEG laboratory of the Centre de Recherche en Neuropsychologie et Cognition (CERNEC, Université de Montréal).

\section{STIMULI}

A video projector placed on the external wall of the shielded MEG room projected the visual stimuli (Presentation V13, Neurobehavioral Systems). Three sets of one hundred pictures were selected from IAPS: unpleasant $(U)$, pleasant $(P)$, and neutral $(N)$. Given the differences usually observed between males and females (Bradley et al., 2001b), pictures were selected according to gender. To control the picture selection, we used linear and quadratic contrasts to compare IAPS standardized valence and arousal ratings (a priori ratings). Linear contrasts on valence ratings (on a scale ranging from 0 , very unpleasant, to 9, very pleasant) were used to compare unpleasant and pleasant categories, and quadratic contrasts on arousal ratings (ranging from 0 , very calm, to 9, very arousing) to compare emotional categories (unpleasant and pleasant) with the neutral category (Hot et al., 2006). Unpleasant $(U)$ and pleasant $(P)$ pictures differed in terms of valence (women: means, $U=2.41, N=5.01$, $P=7.08$, linear contrast, $F(1,297)=3245.06 ; p=6.66 \mathrm{E}-162 ;$ men: $U=2.45, N=4.97, P=7.03$, linear contrast, $F(1,297)=5183.62$; $p=4.62 \mathrm{E}-190$; Figures 1A,B) but were equally arousing, and had higher arousal ratings than neutral $(N)$ pictures (women: means, $U=5.75, N=3.08, \quad P=5.81$, quadratic contrast, $F(1,297)=1372.77 ; p=2.22 \mathrm{E}-113 ;$ men: means, $U=5.64$, $N=2.89, \quad P=5.85 ;$ quadratic contrast, $F(1,297)=1189.65$; 


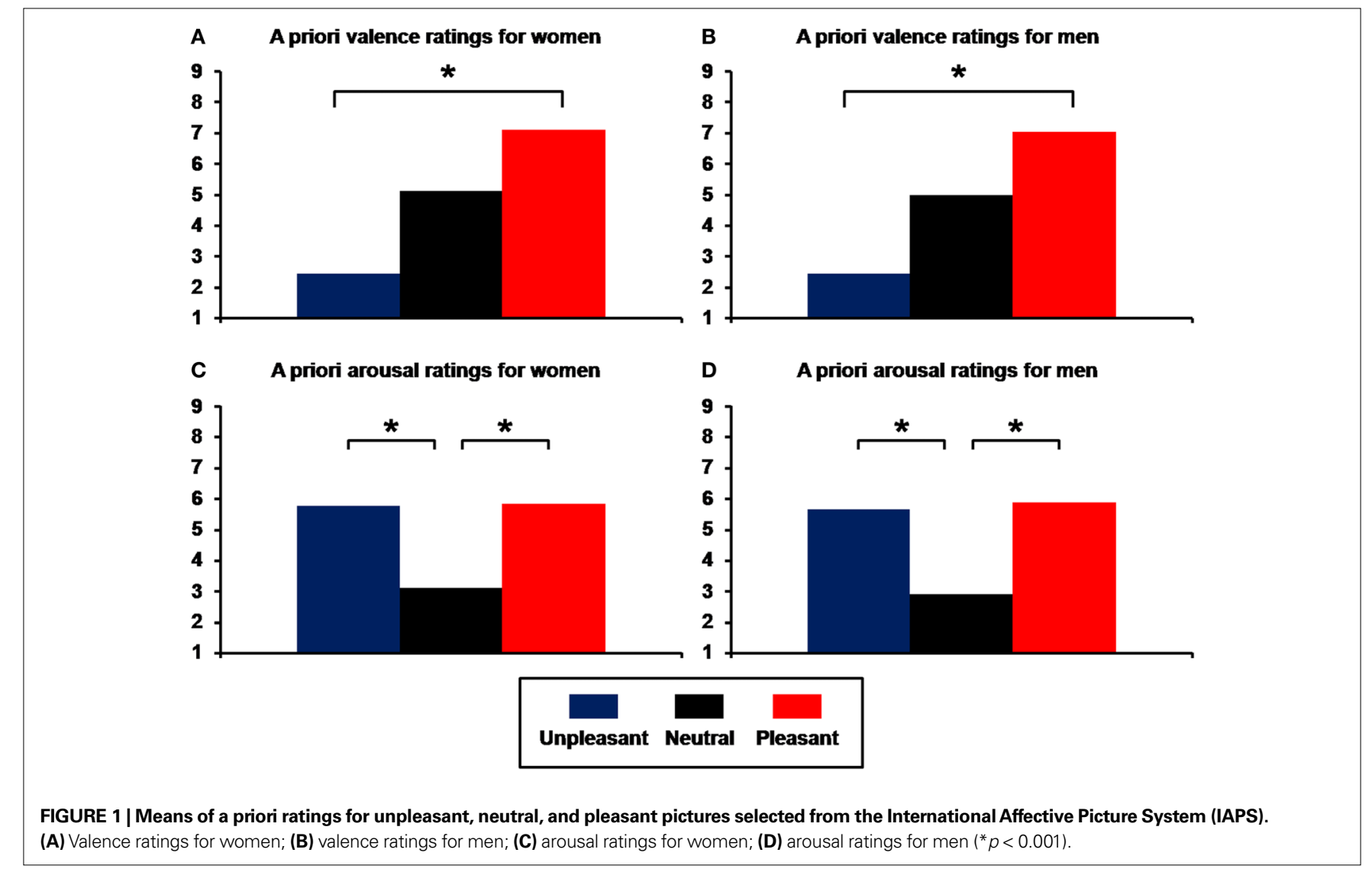

$p=6.99 \mathrm{E}-106$; Figures 1C,D). Additionally, there was no difference in arousal or valence between picture sets shown to men and women (all $F s(1,198)<0.60$ and $p s>0.22)$.

To control for semantic content, each set contained the same number of pictures depicting faces, animals, objects, landscapes, and human beings. Images had an angular size of $12^{\circ}$ (horizontal) and $8^{\circ}$ (vertical) and were homogenized (Image J Software) in terms of main physical characteristics: mean luminance, standard deviation of luminance (i.e., contrast index), and color saturation (red, green, blue). ANOVAs performed on these characteristics did not reveal any difference between unpleasant, neutral, and pleasant scenes in the picture sets shown to men and women (all $F s(2,297)<2.45$ and $p s>0.09)$. Moreover, there was no difference with respect to these features between men and women (all $F s(1,198)<2.52$ and $p s>0.11)$.

\section{RECORDINGS}

SCRs were recorded using the constant-voltage method $(0.5 \mathrm{~V})$ at a sampling rate of $600 \mathrm{~Hz}$, and all trials were low-pass filtered at $50 \mathrm{~Hz}$. $\mathrm{Ag}-\mathrm{AgCl}$ electrodes $(8 \mathrm{~mm}$ diameter active area) filled with $0.05-\mathrm{M} \mathrm{NaCl}$ electrolyte were attached to the palmar side of the middle phalanges of the second and third fingers of participants' hands. Electrodes were connected to Coulbourn S71-23 isolated skin conductance couplers linked to a common acquisition system (CTF MEG 275, CTF Systems) for amplification, storage, and analysis. A sensor was attached to the ring finger of each hand to allow continuous record- ing of skin temperature, known to affect several SCR parameters (Ba-M'hamed et al., 1986; see Materials and Methods in Supplementary Material).

Magnetic fields were measured with a 275-channel whole head magnetometer (CTF MEG 275, CTF Systems) at a sampling rate of $600 \mathrm{~Hz}$. Before and after each experimental session, the position of the participant's head relative to the 275 MEG sensors was located using three fiducial coils placed on the nasion and near the left and right ear. Vertical and horizontal electro-oculograms were also recorded (VEOG-HEOG).

\section{PROCEDURE}

The experimental procedure comprised three steps. In the first step (A), SCRs and MEG data were gathered during the presentation of 60 images (20 unpleasant, 20 neutral, and 20 pleasant) in pseudo-random order (not more than two successive pictures of the same valence). During each trial, a central fixation cross was presented for between 500 and $1500 \mathrm{~ms}$, alerting the participant to the imminent arrival of the picture, which was projected for $500 \mathrm{~ms}$. To avoid SCR habituation effects, the interval between two successive stimuli was randomly varied between 15 and $25 \mathrm{~s}$. Participants were told to try to feel the emotion that was depicted in the pictures. The second step (B) was to increase the MEG signal quality by presenting 240 new IAPS pictures ( 80 unpleasant, 80 neutral, and 80 pleasant) adjusted to those of stage (A) for each emotional and physical characteristic mentioned above (see Stimuli Section) and projected for $500 \mathrm{~ms}$ each, in pseudo-random order, 
with an inter-stimulus interval varying randomly between 2.5 and $3.5 \mathrm{~s}$. This step was divided into four blocks of 60 stimulations (B1, $\mathrm{B} 2, \mathrm{~B} 3$, and B4) to provide the same number of pictures in each experimental block. Finally, in the third step (C), participants had to rate the arousal and valence dimensions of pictures presented in the first two steps using two five-point scales adapted from the Self-Assessment Manikin (SAM; Bradley and Lang, 1994): a valence scale (from very unpleasant to very pleasant) and an arousal scale (from very calm to very arousing).

\section{DATA ANALYSIS}

Participants ratings of valence and arousal were recorded for each presented picture to ensure that the emotional impact on each participant was consistent with IAPS standardized ratings. Thus, a concordance ratio was calculated for each picture using Pearson's coefficient between IAPS standardized arousal and valence ratings and participants' subjective assessments, i.e., between a priori and a posteriori ratings.

To determine SCRs, we used the usual latency criterion of between 1 and $4 \mathrm{~s}$. Trials for which the stimulation did not produce SCRs were nonetheless considered in the averaging data for each participant. To standardize the distribution of SCR magnitude observed in response to stimulation, a logarithmic transformation was conducted according to the formula $\log (1+$ magnitude $)$ (Dawson et al., 2000). A Greenhouse-Geisser corrected repeated measures ANOVA was applied to SCR ratings, with emotional content (unpleasant, neutral, and pleasant) as a within-subject factor. Linear and quadratic contrasts were used to evaluate valence (unpleasant vs. pleasant) and arousal (emotional vs. neutral) effects (Hot et al., 2006), respectively. Importantly, for these physiological recordings we defined the specific arousal effect as a significant difference revealed by quadratic contrast associated with an absence of significant difference indicated by linear contrast. Significance was set at 0.05 (two-tailed).

For MEG recordings, all trials for which the sensor position differed by more than $5 \mathrm{~mm}$-indicating head movement-were removed from the analysis. Third-order gradient noise reduction (computed with CTF software) was applied to MEG signals, which were then baseline-corrected based on mean activity during the $100 \mathrm{~ms}$ prior to the onset of visual stimuli. Trials were lowpass filtered at $35 \mathrm{~Hz}$ and epochs with eye blinks or excessive eye movements were excluded from the analysis by visual inspection of VEOG and HEOG traces (DataEditor, CTF Systems). Finally, averaged event-related magnetic fields (ERFs) were calculated over a period of $700 \mathrm{~ms}$ for each emotional condition (unpleasant, neutral, and pleasant), each experimental block (A, B1, B2, B3, and B4), each 275 MEG sensor, and each participant.

We performed a spatiotemporal principal component analysis (stPCA) on the neuromagnetic data in order to synthesize the spatial and temporal observations on the basis of variability and without a priori hypotheses (Spencer et al., 1999, 2001). Briefly, PCA determines relationships between large numbers of dependent variables and provides components that summarize these relationships. In electrophysiological studies using evoked paradigms, this data-driven analysis has proven to be a powerful descriptive tool to address the complexity and richness of electric fields generated by the human brain recorded by a large number of scalp sensors at high time resolution (Pourtois et al., 2008). Interestingly, this statistical tool has been effectively used in studies investigating the spatiotemporal dynamics of visual emotion perception (Delplanque et al., 2004; Hot et al., 2006; Rigoulot et al., 2008; see Pourtois et al., 2008 for a review).

stPCA consists of two successive PCAs computed on MEG waveforms, providing scores that reflect ERF amplitude at specific localizations (spatial factors, or SFs, extracted during spatial PCA) and specific latencies (temporal factors, or TFs, extracted during temporal PCA) in response to emotional and neutral pictures (detailed EEG procedures are reported in Spencer et al., 2001).

In a first step, we performed a spatial PCA (sPCA) with MEG sensors (275) as dependent variables and time points, emotional conditions, experimental blocks, and participants as observations. The sPCA identifies groups of highly correlated sensors and redistributes them into a smaller number of linear combinations (Varimax rotation, SPSS V. 15 software) called spatial factors (SFs) (Pourtois et al., 2008). Each SF represents a specific spatial configuration of brain activation, and the factor loading corresponds to the SF's contribution to the original variables (i.e., how much the spatial factor accounts for the magnetic field recorded at each sensor). These spatial configurations can be visualized by topographic maps of factor loadings (Cartool software, Denis Brunet) $)^{1}$, and are usually defined by considering sensors with the highest factor loadings (Rigoulot et al., 2008). In addition, sPCA scores reflect the contribution of each SF to each independent variable - i.e., specific time points, emotional conditions, and experimental blocks-for each participant. These factor scores indicate the contribution of the SF to the magnetic fields observed for each original waveform, and can be analyzed by regular statistical tests (Pourtois et al., 2008).

SF scores were subsequently considered as "virtual" ERFs, and their corresponding time series (420 time points, $700 \mathrm{~ms}$ duration at $600 \mathrm{~Hz}$ sampling rate) were subjected to temporal PCA (tPCA), with emotional conditions, experimental blocks, and participants as observations. tPCA identifies groups of highly correlated points in time and redistributes them into new linear combinations (Varimax rotation, SPSS V. 15) called temporal factors (TFs) (Pourtois et al., 2008). tPCA loadings correspond to the TF's contribution to each SF at each time point. Thus, TFs determine SF activity at specific latencies, usually by taking into account the highest factor loadings (Rigoulot et al., 2008).

Finally, the complete stPCA procedure results in a set of factor scores, used here to compare the activity of cerebral configurations (i.e., SFs) at specific latencies (i.e., for each TF) in response to unpleasant, neutral, and pleasant pictures. SFs and TFs were selected for these comparisons using the scree test (Cattell, 1966), a widely used method whereby the curve of decreasing eigenvalues is observed to determine the point where the slope of the curve changes from a rapid, decelerating decline to a flat gradual shape. Only the factorial axes located before this slope decline are retained for further analysis.

A number of control analyses were also performed. First, we verified whether the ISI difference between stages A and B would produce differences in neuromagnetic components showing emotional effect.

${ }^{1}$ http://brainmapping.unige.ch/Cartool.htm 

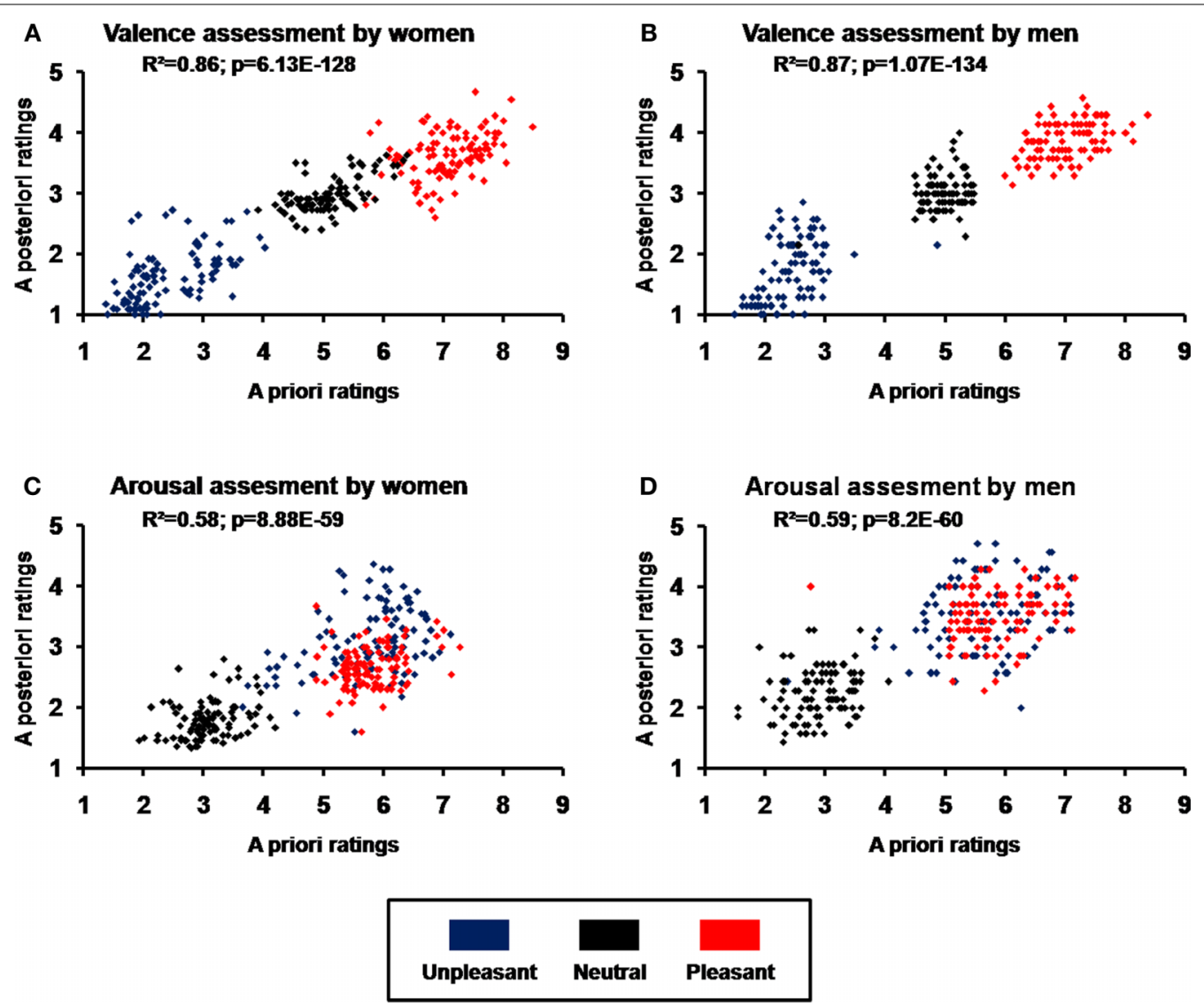

FIGURE 2 |Validation of picture selection using correlations between standardized (a priori) ratings by the International Affective Picture System (IAPS) and mean subjective (a posteriori) ratings of unpleasant, neutral, and pleasant images. (A) Valence ratings for women; (B) valence ratings for men; (C) arousal ratings for women; (D) arousal ratings for men.

For each SF and TF pair, we conducted a corrected GreenhouseGeisser repeated measures ANOVA on individual scores, with emotional conditions (unpleasant, neutral, pleasant) and experimental blocks (A, B1, B2, B3, B4) as within-subject factors. We then verified for SF and TF pairs showing significant effects on emotional conditions ( $p<0.05$, two-tailed) that there was no significant interaction between experimental block and emotional condition. Second, as for the SCR measures, we used linear and quadratic contrasts to evaluate valence and arousal effects, respectively.

To determine the relationship between cerebral and body activations induced by emotional information, we sought to establish correlations between MEG signal characteristics and SCR magnitude. Considering that SCR magnitude constitutes a robust somatovisceral indicator of emotional arousal, the MEG analysis focused on neuromagnetic components showing a specific emotional arousal effect (assessed by a significant quadratic contrast combined with the absence of a significant linear contrast). We then calculated the arousal values, i.e., the activity difference between emotional conditions (mean of pleasant and unpleasant) and the neutral condition assessed by SCR magnitude at stage A and by factor scores at stages $\mathrm{A}$ and $\mathrm{B}$ for neuromagnetic components. Spearman's rank test was then applied to correlate the two emotional measures (SCR and MEG).
Finally, we performed a source reconstruction of MEG activity to determine the cortical areas associated with emotional arousal activity. Cortical current density was mapped by minimum-norm analysis with standard Tikhonov regularization using BrainStorm software (Baillet et al., 2001), which is documented and freely available for download online under the GNU General Public License ${ }^{2}$. Source reconstruction was constrained to the cortical mantle of a generic brain model extracted from the standard brain "MNI/Colin27" defined by the Montreal Neurological Institute. For each participant, surface head models were computed with an overlapping spheres model (Huang et al., 1999). Scalp data used for source reconstruction were the individual arousal values (i.e., the mean of pleasant and unpleasant conditions minus the neutral condition). We applied a Tikhonov regularization parameter $\lambda$ of 0.01 across all participants. We then applied a z-score procedure to the source reconstruction with a $100 \mathrm{~ms}$ baseline before stimulus onset. In order to compare activity revealed by the source reconstruction between the left and right hemispheres, we used functional scout images (Brainstorm software). Scouts correspond to regions of interest defined on the cortical surface, consisting of a subset of surface vertices. We thus

${ }^{2} \mathrm{http}: / /$ neuroimage.usc.edu/brainstorm 
obtained the mean activity of a large pre-defined region. We placed these scouts where we found activity after computing the minimum norm solution. Defined regions in the right hemisphere were as large as in the left hemisphere. We then conducted a corrected GreenhouseGeisser repeated measure ANOVA on individual arousal values with hemispheres (left, right) as a within-subject factor.

\section{RESULTS}

\section{SUBJECTIVE DATA}

Selected pictures were validated by comparing a priori emotional and neutral ratings of valence and arousal and a posteriori subjective mean ratings of the same pictures. Pearson's coefficients revealed significant correlations for both women and men in valence (women, $r(298)=0.92, p=6.1 \mathrm{E}-128$; men, $r(298)=0.93$; $p=1.1 \mathrm{E}-134$; Figures 2A,B) and arousal (women, $r(298)=0.76$, $p=8.9 \mathrm{E}-59$; men, $r(298)=0.77, p=8.2 \mathrm{E}-60$; Figures $2 \mathrm{C}, \mathrm{D})$.

\section{BODY AND BRAIN DATA \\ SCR magnitude}

The SCR analysis revealed higher magnitude for pleasant and unpleasant than for neutral pictures (quadratic contrast, $F(1,17)=12.38, p=0.003$ ), but no significant difference between unpleasant and pleasant pictures (linear contrast, $F(1,17)=1.77$, $p=0.200$; Figure 3).

\section{MEG analysis}

sPCA applied to the $275 \mathrm{MEG}$ sensors yielded 22 SFs that described $96.07 \%$ of the spatial variance in the data set. tPCA was then used to synthesize temporal dimensions of the data set from 420 time points to 29 TFs, which accounted for $97.95 \%$ of the variance. Using the scree test (Cattell, 1966), 6 SFs (accounting for $73.57 \%$ of the variance; Figure $4 \mathrm{~A}$ ) and $4 \mathrm{TFs}$ (accounting for $79.53 \%$ of the variance; Figure 4B) were selected for analysis of variance.

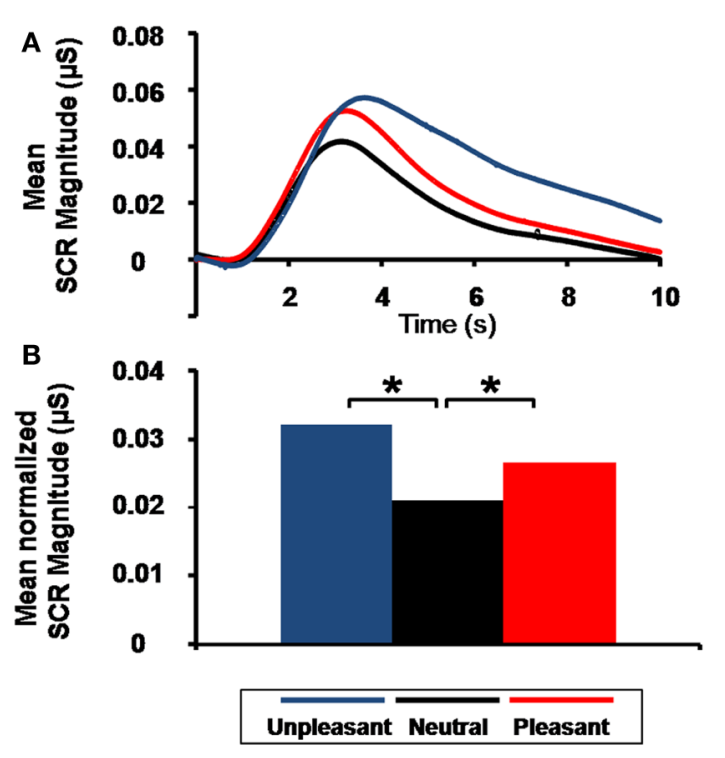

FIGURE 3 | Emotional arousal effect on skin conductance response (SCR). (A) Grand average of SCR as a function of time and emotional content. (B) Mean normalized SCR magnitude as a function of emotional content $\left(^{*} p=0.003\right.$ ).
For each SF and TF pair showing an emotional effect $(p<0.05$, two-tailed), emotion-triggered neuromagnetic activity did not differ between experimental blocks, as the ANOVA indicated no significant interaction between blocks and emotional content (all $F s(8,136)<1.58$ and $p s>0.17)$. We then performed contrast analyses, which revealed greater activity at $180 \mathrm{~ms}$ (indicated by the maximum factor loading for TF3, which accounts for $6.05 \%$ of the variance) in the occipitotemporal component (indicated by the maximum factor loadings for SF3, which account for $8.56 \%$ of the variance) for emotional pictures (unpleasant and pleasant) than for neutral counterparts (quadratic contrast, $F(1,17)=10.70, p=0.004$; Figure 5), with no significant difference between emotional valence (linear contrast, $F(1,17)=1.37, p=0.260)$. No specific arousal effect was found for other neuromagnetic components.

\section{CORRELATED BRAIN-BODY DATA}

Spearman's coefficient calculated on the arousal measures revealed a significant correlation between scores at $180 \mathrm{~ms}$ for the occipitotemporal component (i.e., TF3 for SF3) and SCR magnitude $(\sigma(16)=0.53, p=0.030$; Figure 6A). Thus, the greater the acti-
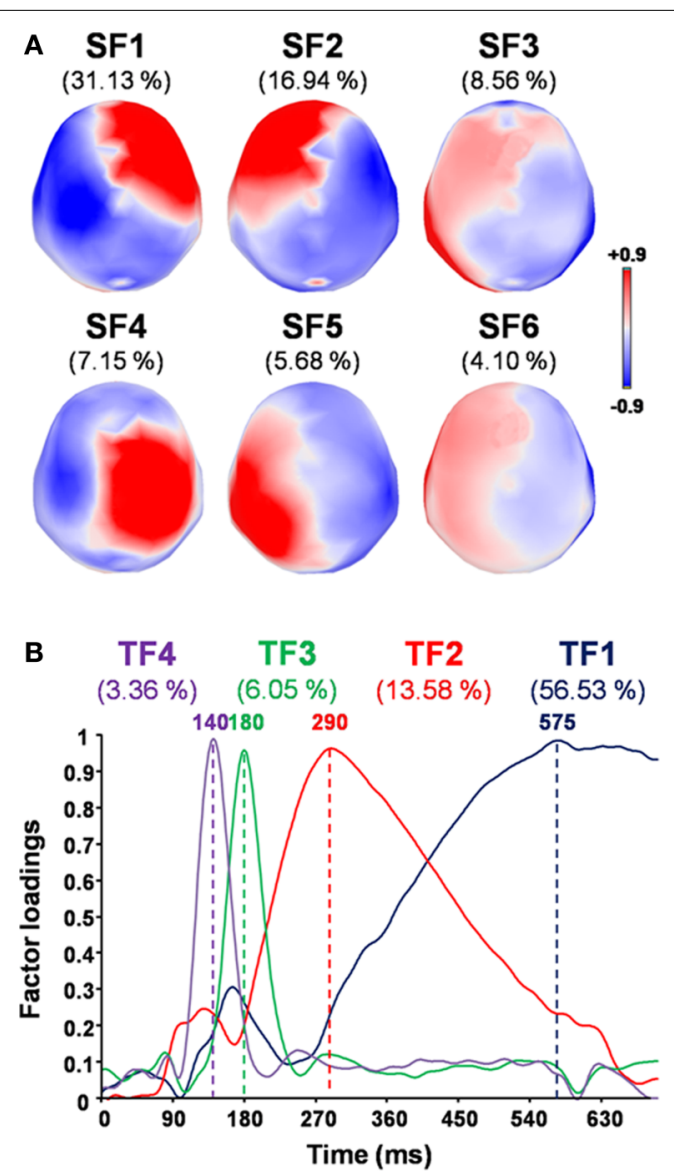

FIGURE 4 | Factor loadings for the selected SFs andTFs, and the corresponding percentage of variance accounted for by each factor. (A) Topographic maps of factor loadings for the selected spatial factors (SFs) as recorded by MEG sensors. (B) Factor loadings of the selected temporal factors (TFs) as a function of time. 


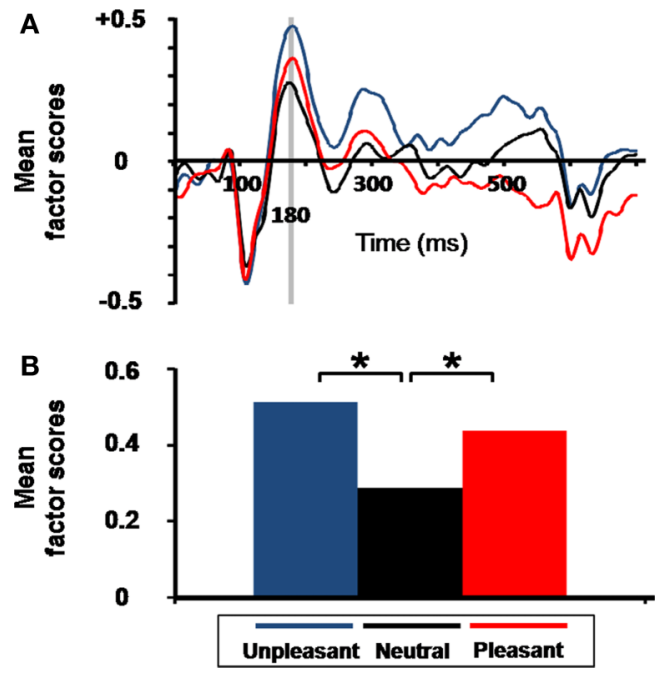

FIGURE 5 | Emotional arousal effect on virtual event-related magnetic field (ERF). (A) Grand average of the mean factor scores of the occipitotemporal component (SF3) as a function of time and emotional condition. Gray bar indicates the latency (revealed by TF3 extracted from temporal PCA), for which a significant emotional arousal effect was observed. (B) Mean factor scores of TF3 (180 ms latency) for SF3 (occipitotemporal component) as a function of emotional content $\left({ }^{*} p=0.004\right)$. Factor scores (y axis) are unitless dimensions.

vation in occipitotemporal areas for a specific individual by the emotional compared to the neutral pictures, the greater the bodily response for this person. Figure 6B shows the cerebral counterpart of this correlation as current density maps of the grand average of the source reconstruction results. At $180 \mathrm{~ms}$, the activation pattern suggests the implication of bilateral occipitotemporal cortical areas, which tend to be more pronounced in the right hemisphere $(F(1,17)=3.20, p=0.091)$.

\section{DISCUSSION}

We used an original method of recording SCRs and ERFs to measure brain activity and bodily responses during affective visual processing. We carefully controlled the emotional categorization of stimuli and applied stPCA to MEG for the first time to identify the spatiotemporal parameters of the data. Statistical analyses revealed that an early difference in visual activity in the occipitotemporal areas between emotional and neutral pictures was significantly correlated with the subsequent impact of emotional arousal at body level.

First, significant correlations between a priori and a posteriori ratings of IAPS pictures in terms of arousal and valence validated our categorization of emotions between unpleasant, neutral, and pleasant pictures for each gender. This subjective feeling assessment confirmed that visual stimulations induced similar reactions in our participants, and that these pictures were appropriate for studying related brain-body correlates. In particular, unpleasant and pleasant pictures were equally arousing and produced a robust emotion-driven body effect, as revealed by the analysis of SCR magnitude. This confirms the utility of SCRs as a reliable measure of autonomic arousal during emotional processing and its impact at body level (Sequeira et al., 2009).
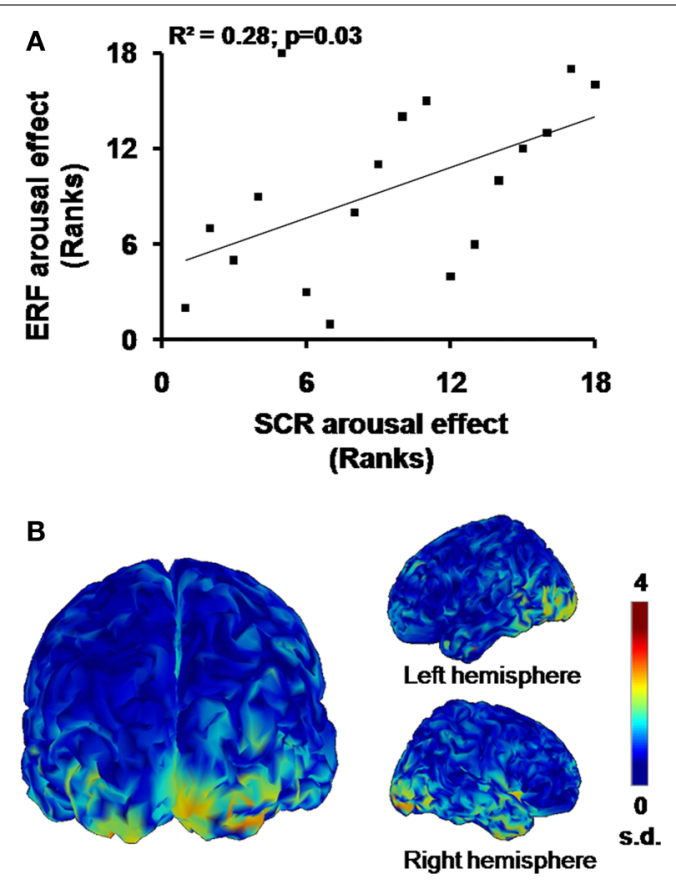

FIGURE 6 | Emotional arousal effects. (A) Significant rank correlation for arousal effects between SCR and ERF. (B) Cortical maps of the grand average ( $n=18$ ) of normalized current dipole densities over the bilateral occipitotemporal areas.

Second, based on a categorization of emotion-eliciting pictures, stPCA applied to MEG data proved to be an efficient means of analyzing and effectively representing both spatial and temporal dimensions of ERFs, as has been previously shown for ERPs (Pourtois et al., 2008). The analysis of variance applied to the results of this procedure revealed greater activity at $180 \mathrm{~ms}$ in an occipitotemporal component for emotional than for neutral pictures, with no difference between emotional valence ( pleasant vs. unpleasant). Previous studies have found enhanced activity in occipitotemporal components in a time window ranging from 120 to $240 \mathrm{~ms}$ for emotional compared to neutral pictures, reflecting activity in the visual processing stream (ERPs, Schupp et al., 2003; Junghöfer et al., 2006; Flaisch et al., 2008; ERFs, Peyk et al., 2008). Activity in the visual cortex in response to arousing emotional scenes extracted from the IAPS has also been observed in neuroimaging studies (Lang et al., 1998; Lane et al., 1999; Paradiso et al., 1999; Bradley et al., 2003; Liberzon et al., 2003; Mourão-Miranda et al., 2003; Sabatinelli et al., 2005; Britton et al., 2006). Taken together, these results suggest that visual activity along the ventral stream is affected by emotional arousal, which constitutes new evidence that emotional cues produce enhanced sensory processing (see Vuilleumier, 2005 for a review). This enhanced visual processing would contribute to the modulation of selective attention as a function of the arousal dimension-for both pleasant and unpleasant visual stimulation-rather than for a specific valence (i.e., for unpleasant or pleasant stimulations only; Vogt et al., 2008).

Third, although previous neuroimaging studies (Fredrikson et al., 1998; Critchley et al., 2000) and one electrophysiological study of SCRs (Keil et al., 2008) have suggested a link between 
visual cortical activity and SCR generation, none has identified the cerebral temporal dynamics of the bodily impact of these emotions. We found an arousal effect at $180 \mathrm{~ms}$ on the occipitotemporal component that was significantly correlated with the arousal effect observed on SCRs (see Figure 6A). This finding leads to two conclusions. The first is that emotional arousal similarly modulates early visual activity along the ventral stream and subsequent body responses controlled by the sympathetic nervous system. This is strongly in favor of a link between both responses. But it also indicates that the amplitude of the central and peripheral emotional responses could depend on individual personality traits: some subjects respond weakly (both peripherally and centrally) to emotionally laden stimulation while other show strong activations, as measured with our indices. Second, source analyses suggest predominant right hemisphere activation in response to emotional stimuli, which corroborates previous observations of arousal emotional processing and autonomic activation (Spence et al., 1996; Lane et al., 1999).

Hypothetically, the brain-body arousal effect highlighted in this study could result from the rapid detection of emotional content by the amygdala (Morris et al., 1998; Davidson and Irwin, 1999; Vuilleumier, 2005). Indeed, the amygdala plays a crucial role in both perceiving emotional cues and producing emotional responses (Davidson and Irwin, 1999). First, amygdalar connections to the occipitotemporal cortices have been extensively described (Amaral et al., 2003), as well as their impact on visual activity during emotional processing (Morris et al., 1998; Pessoa et al., 2002; Sabatinelli et al., 2005; see Vuilleumier, 2005, for a review). Moreover, amygdalar activity in response to emotional stimuli appears to be sensitive to the arousal dimension of emotional processing rather than to its valence (Sergerie et al., 2008). In fact, similar activations of this key region have been observed in response to both pleasant and unpleasant pictures (Garavan et al., 2001; Liberzon et al., 2003; Sabatinelli et al., 2005; Kensinger and Schacter, 2006). Furthermore, the amygdala concomitantly modulates spinal preganglionic sudomotor neurons via direct amygdalospinal projections or indirectly by reticular structures (Holstege, 1992), both networks playing a central role in triggering SCRs (Sequeira et al., 2009). Direct stimulation of the human amygdala elicits SCRs (Mangina and Beuzeron-Mangina, 1996), lesions of the amygdala result in an abolition of emotionally conditioned SCRs (Bechara et al., 1995), and neuroimaging studies show a correlation between activity in the amygdala and SCR amplitude (Liberzon et al., 2000; Williams et al., 2001). Finally, a recent study using positron emission tomography (PET) showed that activity in the amygdala was linked to SCR occurrence in monkeys (Laine et al., 2009).

A limitation of our study is that by using MEG we were unable to directly test the implication of subcortical structures, in particular amygdala activity. Nevertheless, an argument in favor of the contribution of the amygdala to this brain-body interaction is the existence of a direct short-latency pathway from the thalamus to the amygdala - first demonstrated in animal studies (LeDoux et al., 1985)—allowing the detection of emotional content of visual stimuli without awareness (LeDoux, 1996). Interestingly, SCRs have been observed in response to emotional facial expressions (EFE) conditioned to predict an aversive electrical shock and presented so as to prevent awareness (Öhman, 1992). Furthermore, Silvert et al. (2004) observed that masked and unmasked emotional words elicited greater SCR amplitude than neutral words. In addition, in a neuroimaging study, modulated amygdalar activity was observed in response to masked EFE without explicit knowledge (Whalen et al., 1998). Consistent with this finding, recognition of fearful faces was observed despite a lesion of striate visual cortex similar to that found in a blindsight patient (de Gelder et al., 1999) or a hemianope patient (de Gelder et al., 2005). In the latter case, unconscious perception of facial expression was associated with enhanced activity in the fusiform gyrus, amygdala, and pulvinar (de Gelder et al., 2005).

In light of these results, the arousal dimension of emotional stimuli appears to modulate in a similar way both early cerebral visual activity and later body responses. In a recent study that simultaneously recorded MEG and electrocardiograms, Rudrauf et al. (2009) also observed that body responses to emotional stimuli can occur during the first $500 \mathrm{~ms}$ after visual presentation, and could be represented in the brain even in the early phase of emotional induction. Using SCRs, which have the further advantage of specifically reflecting sympathetic nervous system responses (Critchley, 2009), our results indicate that even though peripheral physiological responses can occur quite early after emotional visual stimuli are presented (Rudrauf et al., 2009), similar brain activations are triggered even earlier. These findings contradict the James-Lange theory of emotion. Nevertheless, this assumption does not imply that peripheral expressions of emotions are generated simultaneously with the subjective feeling, as posited by the Cannon-Bard theory (Damasio et al., 2000). Indeed, the bodily impact of emotions appears to rely on rapid and certainly unconscious detection of the emotional arousal, probably by the amygdala. Feedback from the periphery could in turn participate in the subjective feeling, through bidirectional connections between anterior affective structures and somatosensory and viscerosensory structures (Rudrauf et al., 2009). Finally, according to the "body-loop" proposed by Damasio in his somatic marker hypothesis, the body responses generated mainly by subcortical structures would inform cortical areas of the internal environment state, most certainly at a prefrontal level (Damasio, 1994). In this context, neuroimaging studies have observed associations between medial frontal activity and SCRs in response to fear as depicted in EFE (Williams et al., 2001) and IAPS pictures (Anders et al., 2004). Concomitantly, the enhanced cortical visual processing, certainly mediated through amygdalar connections, would allow a more efficient treatment of emotional stimulation to inform individuals about their external environment (Vuilleumier, 2005). Together, these peripheral and central components of nervous activity would produce a subjective feeling and appropriate behavioral responses (Damasio, 1999). In our study, the fact that occipitotemporal activity is affected in the same way as SCRs constitutes indirect proof of this interaction.

In conclusion, we examined SCR magnitude to determine the temporal course of a neuromagnetic cortical component linked to a bodily expression of emotion. This link suggests that emotional stimuli in the external environment induce a specific mental state involving an early brain-body interaction as a function of emotional arousal. Finally, the emotion-body modulation highlighted in this study provides new insights into the neural bases of complex and reciprocal mind-body links. 


\section{ACKNOWLEDGMENTS}

This work was supported by grants from the Ministère de la Recherche, France (Fabien D'Hondt, Simon Rigoulot), the Rotary Foundation (Fabien D'Hondt), CNRS and the Centre International de Lille 1 (Henrique Sequeira), the Fonds de la Recherche en Santé du Québec (FRSQ) (group grant), the Canada Research Chair Program (Maryse Lassonde, Franco Lepore), the Canadian Institutes of Health Research (Maryse Lassonde, Franco Lepore), and the Natural Sciences and Engineering Research Council of Canada (Maryse Lassonde, Franco Lepore). Olivier Collignon is a

\section{REFERENCES}

Amaral, D. G., Behniea, H., and Kelly, J. L. (2003). Topographic organization of projections from the amygdala to the visual cortex in the macaque monkey. Neuroscience 118, 1099-1120.

Amrhein, C., Muhlberger, A., Pauli, P., and Wiedemann, G. (2004). Modulation of event-related brain potentials during affective picture processing: a complement to startle reflex and skin conductance response? Int. J. Psychophysiol. 54, 231-240.

Anders, S., Lotze, M., Erb, M., Grodd, W., and Birbaumer, N. (2004). Brain activity underlying emotional valence and arousal: a response-related fMRI study. Hum. Brain Mapp. 23, 200-209.

Ba-M'hamed, S., Ciancia, F., Delerm, B., Roy, J. C., and Sequeira-Martinho, A. H. (1986). Influence of skin temperature on latency and amplitude of skin potential responses in the cat. Biol. Psychol. 22, 59-67.

Baillet, S., Mosher, J. C., and Leahy, R. M. (2001). Electromagnetic brain mapping. IEEE Signal Process. Mag. 18, 14-30.

Bechara, A., Tranel, D., Damasio, H., Adolphs, R., Rockland, C., and Damasio, A. R. (1995). Double dissociation of conditioning and declarative knowledge relative to the amygdala and hippocampus in humans. Science 269, 1115-1118.

Bradley, M. M., Codispoti, M., Cuthbert, B. N., and Lang, P. J. (2001a). Emotion and motivation I: defensive and appetitive reactions in picture processing. Emotion 1, 276-298.

Bradley, M. M., Codispoti, M., Sabatinelli, D., and Lang, P. J. (2001b). Emotion and motivation II: sex differences in picture processing. Emotion 1, 300-319.

Bradley, M. M., and Lang, P. J. (1994). Measuring emotion: the selfassessment manikin and the semantic differential. J. Behav. Ther. Exp. Psychiatry 25, 49-59.

Bradley, M. M., Sabatinelli, D., Lang, P. J., Fitzsimmons, J. R., King, W., and Desai, P. (2003). Activation of the visual cortex in motivated attention. Behav. Neurosci. 117, 369-380.

Britton, J.C., Taylor, S. F., Sudheimer, K. D., and Liberzon, I. (2006). Facial expressions and complex IAPS pictures: common and differential networks. Neuroimage 31, 906-919.

Cattell, R. B. (1966). The scree test for the Res. 1, 245-276.

Critchley, H.D. (2009). Psychophysiology of neural, cognitive and affective integration: fMRI and autonomic indicants. Int. J. Psychophysiol. 73, 88-94.

Critchley, H. D., Elliott, R., Mathias, C. J., and Dolan, R. J. (2000). Neural activity relating to generation and representation of galvanic skin conductance responses: a functional magnetic resonance imaging study. J. Neurosci. 20, 3033-3040.

Damasio, A. R. (1994). Descartes' Error: Emotion, Reason, and the Human Brain. (New York: Grosset/Putnam).

Damasio, A. R. (1996). The somatic marker hypothesis and the possible functions of the prefrontal cortex. Philos. Trans. R. Soc. Lond., B, Biol. Sci. 351, 1413-1420.

Damasio, A. R. (1999). The Feeling of What Happens: Body and Emotion in the Making of Consciousness. (New York: Harcourt Brace).

Damasio, A. R. (2000). "A second chance for emotion," in Cognitive Neuroscience of Emotion, eds R. D. Lane and L. Nadel (New York: Oxford University Press), 12-23.

Damasio, A. R., Grabowski, T. J., Bechara, A., Damasio, H., Ponto, L. L., Parvizi, J., and Hichwa, R. D. (2000). Subcortical and cortical brain activity during the feeling of self-generated emotions. Nat. Neurosci. 3, 1049-1056.

Damasio, A. R., Tranel, D., and Damasio, H. C. (1991). "Somatic markers and the guidance of behavior: theory and preliminary testing," in Frontal Lobe Function and Dysfunction, eds H. S. Levin, H. M. Eisenberg and L. B. Benton (New York: Oxford University Press), 217-229.

Davidson, R. J., and Irwin, W. (1999). The functional neuroanatomy of emotion number of factors. Multivariate Behav.

postdoctoral researcher at the Belgian National Funds for Scientific Research. Cartool software was programmed by Denis Brunet of the Functional Brain Mapping Laboratory, Geneva, Switzerland, and is supported by the Center for Biomedical Imaging (CIBM) of Geneva and Lausanne.

\section{SUPPLEMENTARY MATERIAL}

The Supplementary Material for this article can be found online at http://www.frontiersin.org/humanneuroscience/paper/10.3389/ fnhum.2010.00033/

and affective style. Trends Cogn. Sci. $3,11-21$.

Davis, M., and Whalen, P. J. (2001). The amygdala: vigilance and emotion. $\mathrm{Mol}$. Psychiatry 6, 13-34.

Dawson, M.E., Schell,A. M., and Filion, D. L. (2000). "The electrodermal system," in Handbook of Psychophysiology, eds J. T. Cacioppo, L. G. Tassinary and G. G. Bernston (New York: Cambridge University Press), 201-233.

de Gelder, B., Morris, J. S., and Dolan, R. J. (2005). Unconscious fear influences emotional awareness of faces and voices. Proc. Natl. Acad. Sci. U.S.A. 102, 18682-18687.

de Gelder, B., Vroomen, J., Pourtois, G., and Weiskrantz, L. (1999). Nonconscious recognition of affect in the absence of striate cortex. Neuroreport 10, 3759-3763.

Delplanque, S., Lavoie, M. E., Hot, P. Silvert, L., and Sequeira, H. (2004). Modulation of cognitive processing by emotional valence studied through event-related potentials in humans. Neurosci. Lett. 356, 1-4.

Flaisch, T., Stockburger, J., and Schupp, H T. (2008). Affective prime and target picture processing: an ERP analysis of early and late interference effects. Brain Topogr. 20, 183-191.

Fredrikson, M., Furmark, T., Olsson, M. T., Fischer, H., Andersson, J., and Långström, B. (1998). Functional neuroanatomical correlates of electrodermal activity: a positron emission tomographic study. Psychophysiology 35, 179-185.

Friedman, B. H. (2009). Feelings and the body: the Jamesian perspective on autonomic specificity of emotion. Biol. Psychol. doi:10.1016/j.biopsycho.2009.10.006

Garavan, H., Pendergrass, J. C., Ross, T. J., Stein, E. A., and Risinger, R. C. (2001). Amygdala response to both positively and negatively valenced stimuli. Neuroreport 12, 2779-2783.

Grimm, S., Schmidt, C. F., Bermpohl, F., Heinzel, A., Dahlem, Y., Wyss, M., Hell, D., Boesiger, P., Boeker, H., and Northoff, G. (2006). Segregated neural representation of distinct emo- tion dimensions in the prefrontal cortex-an fMRI study. Neuroimage 30, 325-340.

Holstege, G. (1992). The emotional motor system. Eur. J. Morphol. 30, 67-79.

Hot, P., Saito, Y., Mandai, O., Kobayashi, T., and Sequeira, H. (2006). An ERP investigation of emotional processing in European and Japanese individuals. Brain Res. 1122, 171-178.

Huang, M. X., Mosher, J. C., and Leahy, R. M. (1999). A sensor-weighted overlapping-sphere head model and exhaustive head model comparison for MEG. Phys. Med. Biol. 44, 423-440.

Junghöfer, M., Sabatinelli, D., Bradley, M. M., Schupp, H. T., Elbert, T. R., and Lang, P. J. (2006). Fleeting images: rapid affect discrimination in the visual cortex. Neuroreport 17, 225-229.

Keil, A., Smith, J. C., Wangelin, B. C., Sabatinelli, D., Bradley, M. M., and Lang, P. J. (2008). Electrocortical and electrodermal responses covary as a function of emotional arousal: a single-trial analysis. Psychophysiology 45, 516-523.

Kensinger, E. A., and Schacter, D. L. (2006). Processing emotional pictures and words: effects of valence and arousal. Cogn. Affect. Behav. Neurosci. 6, 110-126.

Laine, C. M., Spitler, K. M., Mosher, C. P., and Gothard, K.M. (2009). Behavioral triggers of skin conductance responses and their neural correlates in the primate amygdala. J. Neurophysiol. 101, 1749-1754.

Lane, R. D., Chua, P. M. L., and Dolan, R. J. (1999). Common effects of emotional valence, arousal and attention on neural activation during visual processing of pictures. Neuropsychologia 37, 989-997.

Lane, R. D., Reiman, E. M., Bradley, M. M., Lang, P. J., Ahern, G. L., Davidson, R. J., and Schwartz, G. E. (1997). Neuroanatomical correlates of pleasant and unpleasant emotion. Neuropsychologia 35, 1437-1444.

Lane, R. D., Waldstein, S. R., Chesney, M. A., Jennings, J. R., Lovallo, W. R., Kozel, P. J., Rose, R. M., Drossman, D. A., Schneiderman, N., Thayer, J. 
F., and Cameron, O. G. (2009). The rebirth of neuroscience in psychosomatic medicine, Part I: historical context, methods, and relevant basic science. Psychosom. Med. 71, 117-134.

Lang, P. J. (1994). The varieties of emotional experience: a meditation on James-Lange theory. Psychol. Rev. 101, 211-221.

Lang, P. J., Bradley, M. M., and Cuthbert, B. N. (2005). International Affective Picture System (IAPS): Affective Ratings of Pictures and Instruction Manual. Technical Report A-8. Gainesville, FL: University of Florida.

Lang, P. J., Bradley, M. M., Fitzsimmons, J. R., Cuthbert, B. N., Scott, J. D., Moulder, B., and Nangia, V. (1998). Emotional arousal and activation of the visual cortex: an fMRI analysis. Psychophysiology 35, 199-210.

Lang, P. J., Greenwald, M. K., and Bradley, M. M., and Hamm, A. O. (1993). Looking at pictures: affective, facial, visceral, and behavioral reactions. Psychophysiology 30 261-273.

LeDoux, J.E. (1996). The Emotional Brain. (New York: Simon and Shuster).

LeDoux, J. E., Ruggiero, D. A., and Reis, D. J. (1985). Projections to the subcortical forebrain from anatomically defined regions of the medial geniculate body in the rat. J. Comp. Neurol. 242, 182-213.

Liberzon, I., Phan, K. L., Decker, L. R., and Taylor, S. F. (2003). Extended amygdala and emotional salience: a PET activation study of positive and negative affect. Neuropsychopharmacology 28, 726-733.

Liberzon, I., Taylor, S. F., Fig, L. M., Decker, L. R., Koeppe, R. A., and Minoshima, S. (2000). Limbic activation and psychophysiologic responses to aversive visual stimuli. Interaction with cognitive task. Neuropsychopharmacology 23, 508-516.

Mangina, C.A., and Beuzeron-Mangina, J. H. (1996). Direct electrical stimulation of specific human brain structures and bilateral electrodermal activity. Int. J. Psychophysiol. 22, 1-8.

Morris, J. S., Friston, K. J., Buchel, C., Frith, C. D., Young, A. W., Calder, A. J., and Dolan, R. J. (1998). A neuromodulatory role for the human amygdala in processing emotional facial expressions. Brain 121, 47-57.

Mourão-Miranda, J., Volchan, E., Moll, J., de Oliveira-Souza, R., Oliveira, L., Bramati, I., Gattass, R., and Pessoa, L. (2003). Contributions of stimulus valence and arousal to visual activation during emotional perception. Neuroimage 20, 1955-1963.

Öhman, A. (1992). "Fear and anxiety as emotional phenomena: clinical phenomenology, evolutionary perspectives, and information-processing mechanisms," in Handbook of Emotions, eds M. Lewis and J. M. Haviland (New York: Guilford), 511-536.

Oldfield, R.C. (1971). The assessment and analysis of handedness: the Edinburgh inventory. Neuropsychologia 9, 97-113.

Paradiso, S., Johnson, D. L., Andreasen, N. C., O'Leary, D. S., Watkins, G. L., Ponto, L. L., and Hichwa, R. D. (1999). Cerebral blood flow changes associated with attribution of emotional valence to pleasant, unpleasant, and neutral visual stimuli in a PET study of normal subjects. Am. J. Psychiatry 156, 1618-1629.

Pessoa, L., McKenna, M., Gutierrez, E., and Ungerleider, L. G. (2002). Neural processing of emotional faces requires attention. Proc. Natl. Acad. Sci. U.S.A. 99, 11458-11463.

Peyk, P., Schupp, H. T., Elbert, T., and Junghöfer, M. (2008). Emotion processing in the visual brain: a MEG analysis. Brain Topogr. 20, 205-215.

Phan, K. L., Taylor, S. F., Welsh, R. C., Ho, S. H., Britton, J. C., and Liberzon, I. (2004). Neural correlates of individual ratings of emotional salience: a trialrelated fMRI study. Neuroimage 21, 768-780.

Pourtois, G., Delplanque, S., Michel, C., and Vuilleumier, P. (2008). Beyond conventional event-related brain potential (ERP): exploring the timecourse of visual emotion processing using topographic and principal component analyses. Brain Topogr. 20, 265-277.

Ribeiro, R. L., Teixeira-Silva, F., Pompeia, S., and Bueno, O. F. (2007). IAPS includes photographs that elicit lowarousal physiological responses in healthy volunteers. Physiol. Behav. 91, 671-675.
Rigoulot, S., Delplanque, S., Despretz, P., Defoort-Dhellemmes, S., Honoré, J., and Sequeira, H. (2008). Peripherally presented emotional scenes: a spatiotemporal analysis of early ERP responses. Brain Topogr. 20, 216-223.

Rudrauf, D., Lachaux, J. P., Damasio, A., Baillet, S., Hugueville, L., Martinerie, J., Damasio, H., and Renault, B. (2009). Enter feelings: somatosensory responses following early stages of visual induction of emotion. Int. J. Psychophysiol. 72, 13-23.

Sabatinelli, D., Bradley, M. M. Fitzsimmons, J. R., and Lang, P. J. (2005). Parallel amygdala and inferotemporal activation reflect emotional intensity and fear relevance. Neuroimage 24, 1265-1270.

Schupp, H. T., Junghöfer, M., Weike, A. I., and Hamm, A. O. (2003). Emotional facilitation of sensory processing in the visual cortex. Psychol. Sci. 14, 7-13.

Sergerie, K., Chochol, C., and Armony, J. L. (2008). The role of the amygdala in emotional processing: a quantitative meta-analysis of functional neuroimaging studies. Neurosci. Biobehav. Rev. 32, 811-830.

Sequeira, H., Hot, P., Silvert, L., and Delplanque, S. (2009). Electrical autonomic correlates of emotion. Int. J. Psychophysiol. 71, 50-56.

Silvert, L., Delplanque, S., Bouwalerh, H., Verpoort, C., and Sequeira, H. (2004). Autonomic responding to aversive words without conscious valence discrimination. Int. J. Psychophysiol. 53, 135-145.

Spence, S., Shapiro, D., and Zaidel, E. (1996). The role of the right hemisphere in the physiological and cognitive components of emotional processing. Psychophysiology 33, 112-122.

Spencer, K. M., Dien, J., and Donchin, E. (1999).A componential analysis of the ERP elicited by novel events using a dense electrode array. Psychophysiology 36, 409-414.

Spencer, K. M., Dien, J., and Donchin, E. (2001). Spatiotemporal analysis of the late ERP responses to deviant stimuli. Psychophysiology 38, 343-358.

Taylor, S. F., Phan, K. L., Decker, L. R., and Liberzon, I. (2003). Subjective rating of emotionally salient stimuli modulates neural activity. Neuroimage 18, 650-659.

Vogt, J., De Houwer, J., Koster, E. H., Van Damme, S., and Crombez, G. (2008). Allocation of spatial attention to emotional stimuli depends upon arousal and not valence. Emotion 8, 880-885.

Vuilleumier, P.(2005). How brains beware: neural mechanisms of emotional attention. Trends Cogn. Sci. (Regul. Ed.) 9, 585-594.

Whalen, P. J., Rauch, S. L., Etcoff, N. L., McInerney, S. C., Lee, M. B., and Jenike, M. A. (1998). Masked presentations of emotional facial expressions modulate amygdala activity without explicit knowledge. J. Neurosci. 18, 411-418.

Williams, L. M., Phillips, M. L., Brammer, M. J., Skerrett, D., Lagopoulos, J., Rennie, C., Bahramali, H., Olivieri, G., David, A. S., Peduto, A., and Gordon E. (2001). Arousal dissociates amygdala and hippocampal fear responses: evidence from simultaneous fMRI and skin conductance recording. Neuroimage 14, 1070-1079.

Conflict of Interest Statement: The authors declare that this study was conducted in the absence of any commercial or financial relationships that could be construed as a potential conflict of interest.

Received: 14 October 2009; paper pending published: 17 December 2009; accepted: 31 March 2010; published online: 19 April 2010.

Citation: D'Hondt F, Lassonde M, Collignon $O$, Dubarry A-S, Robert M, Rigoulot S, Honoré J, Lepore F and Sequeira H (2010) Early brain-body impact of emotional arousal. Front. Hum. Neurosci. 4:33. doi: 10.3389/fnhum.2010.00033

Copyright (C) 2010 D'Hondt, Lassonde, Collignon, Dubarry, Robert, Rigoulot, Honoré, Lepore and Sequeira. This is an open-access article subject to an exclusive license agreement between the authors and the Frontiers Research Foundation, which permits unrestricted use, distribution, and reproduction in any medium, provided the original authors and source are credited. 